\title{
Über geschlossene Extremalen und eine einfache Herleitung der isoperimetrischen Ungleichungen.
}

\author{
Memoria di JoHann Radon (a Wien).
}

Zusammenfassung. - Unter den zahlreichen Beweisen, die für die isoperimetrische Eigenschaft des Kreises und verwandte Ungleichungen gegeben worden sind, erfreuen sich diejenigen, die sich der Methoden der Variationsrechnung bedienen, im allgemeinen keiner besonderen Beliebtheit, da sie verhältnismässig kompliziert sind. T. BONNESEN (l) hat in einer bekannten Arbeit gezeigt, wie man mit Methoden der Variationsrechnung aum Ziel kommt, ohne die Theorie des isoperimetrischen Problens im eigètlichen Sinn zu benützen und ist sogar bis au wesentlichen Verschärfungen der zu beweisenden Ungleichungen gelangt.

Wenn ich im folgenden mit einer neuen Methode, die übrigens mit der von BoNNESEN nahe verwandt ist, auf das alte Problem zurückkomme, so geschieht dies aus dem Grunde, dass mir diese Methode besonders einfach erscheint und zu Beweisen führt, die sich. ohne die technischen Hilfswittel der Variationsrochnung leicht durchfuhren lassen (diesbezüglich sei auf den letzten Teil der Arbeit verwiesen). Ferner trägt meine Methode insofern sehr weit, als sie ohne weiters auf den nichteuklidische Fall anwendbar. ist, Konvexität nicht voraussetat und vermutlich auch in anderen ähnlichen Eällen zum Ziel fuhrt.

I.

1. Wir beginnen mit einigen Betrachtungen ubber das einfachste Variationsproblem in Parameterdarstellung.

$$
J=\int_{t_{0}}^{t_{1}} F(x, y, \dot{x}, \dot{y}) d t
$$

sei das zum Extrem zu machende Integral.

Eine einparametrige Extremalenschar

$$
x=x(t, a), y=y(t, a), \quad t_{0} \leqq t \leqq t_{1}, A_{0} \leqq a \leqq A_{1}
$$

gestattet bekanntlich jeden einfachen, ihr angehörenden Extremalenbogen längs dessen die Funktionaldeterminante

$$
\Delta=\frac{\partial x, y)}{\partial(t, a)}
$$

ungleich Null ist, in ein Feld einzubetten. Dort sind die beiden Ableitungen

$$
F_{\dot{x}}(x, y, \dot{x}, \dot{y}), \quad F_{\dot{y}}(x, y, \dot{x}, \dot{y})
$$

(1) "Acta Mathematica ", Bd. 48 (1926). 
als Funktionen des Ortes betrachtet, die partiellen Ableitnngen einer Funk tion $W(x, y)$ :

$$
F_{\dot{x}}=W_{x}, \quad F_{\dot{y}}=W_{y}
$$

(Unabhängigkeittsatz von HLLBERT). Verlängern wir einen Extremalenbogen des Feldes nach beiden Seiten bis zu den «Brennpunkten» $A_{1} A_{2}, d, h$. bis zu den ersten Punkten, in denen $\Delta=0$, so treffen wir dort die Einhullende der Schar $\left(H_{1}\right.$ bzw. $H_{2}$, sieehe Figur 1). Es steht nun niehts im Wege, die

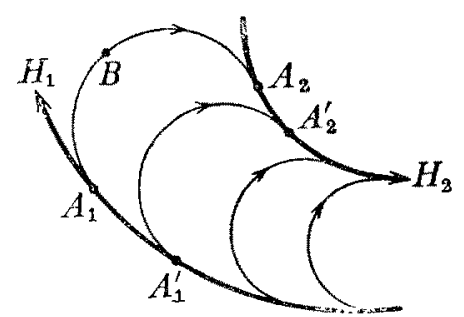

Fig. 1.

Funktion $W$ auch noch auf $H_{1}$ und $H_{2}$ zu definieren, indem man z.B. ihren Wert in $A_{2}$ durch

$$
W_{A_{2}}=W_{B}+J_{B A_{2}}
$$

erklärt, wobei das Integral $J$ längs des Extremalenbogens $B A_{2}$ unserer Schar zu nehmen ist. $W$ ist dann im ganzen «uneigentlichen 》 Feld, das durch die Hullen $H_{1}, H_{2}$ und etwa $a=a_{1}, a=a_{2}$ begrenzt zu denken ist, stetig. Beachtet man jetzt, dass auch auf den beiden Hüllen durch die Orientierung der sie berührenden Extremalenbogen (im Sinn wachsender $t$ ) eine Orientierung gegeben ist, (den Fall singulärer Punkte, insbesondere Spitzen der Hüllen schliessen wir ausdrücklich aus), so gilt nach dem Hüllensatz von A. KNESER:

$$
W_{A_{2}^{\prime}}-W_{A_{2}}=J_{A_{2} A^{\prime}{ }_{2}}
$$

unter der Voraussetzung, dass $A_{2}$ auf $H_{2}$ ror $A_{2}^{\prime}$ liegt und das Integral $I_{A_{2} A_{2}}$ längs $H_{2}$ genommen wird, Entsprechendes gilt für $H_{1}$.

2. Wir nehmen jetzt an, dass es eine einparametrige Sohar geschlossener Extremalen $E$ gibt, die zwischen zwei Hüllkurven $H_{1}$ and $H_{2}$ derart verlaufen, dass bei Beschränkung auf die Teilbogen $A_{1} A_{2}$ bzw. $A_{2} A_{1}$ (Fig. 2) jedesmal

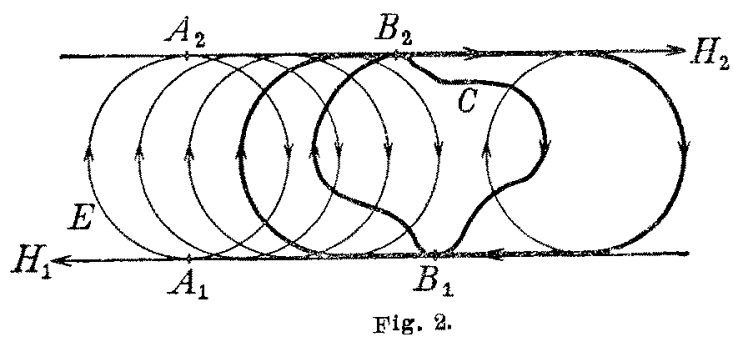


ein uneigentliches Feld im Sinn von 1 . entsteht. Es ist klar, dass $J$ fur alle Extremalen $E$ bei einmaligem Umlauf denselben Wert hat.

Zu jedem der so entstehenden Felder gehört eine Funktion $W$ bzw. $W^{\prime}$ und nach (1) ist die Differenz $W-W^{\prime}$ auf jeder der Hullen konstant.

Als «Feld" werde von jetzt ab das Gebiet zwischen $H_{1}$ und $H_{2}$ bezeichnet, soweit es beiden uneigentlichen Feldern angehört.

Betrachten wir jetzt eine beliebige geschlossene Kurve $C$, die ganz im Feld verlänft und $H_{i}$ in $B_{i}$ trifft $(i=1,2)$. Es ist :

$$
\begin{aligned}
& J_{C}=I_{B_{2} B_{2}}+J_{B_{2} B_{1}} \\
& J_{B_{1} B_{2}}-\left(W_{B_{2}}-W_{B_{1}}\right)=\int_{t_{0}}^{t_{1}}\left(F-W_{x} \dot{x}-W_{y} \dot{y}\right) d t \\
& J_{B_{2} B_{1}}-\left(W_{B_{1}}^{\prime}-W_{B_{2}}^{\prime}\right)=\int_{t_{1}}^{t_{2}}\left(F-W^{\prime}{ }_{x} \dot{x}-W^{\prime}{ }_{y} \dot{y}\right) d t .
\end{aligned}
$$

Der Integrand rechts ist beidemale die WEIERsTrass'sche E-Funktion; sind also die Extremalen stark, as sind die Integrals beide $\geqq 0$.

Also folgt durch Addition:

$$
J_{C} \geqq\left(W_{B_{2}}-W_{B_{2}}^{\prime}\right)-\left(W_{B_{1}}-W_{B_{1}}^{\prime}\right)=\left(W_{A_{2}}-W_{A_{2}}^{\prime}\right)-\left(W_{A_{1}}-W_{A_{1}}^{\prime}\right) \text {. }
$$

Die rechte Seite stellt aber den Wert von $J$ längs einer der geschlossenen Extremalen dar. Also hat man:

$$
J_{C} \geqq J_{E} .
$$

Wenn hier das Gleichheitszeichen gelten soll, so müssen die beiden E-Funktionen Null sein, d.h., die Richtung von $C$ muss mit der Feldrichtung iibereinstimmen. Das bedeutet, dass der Bogen $B_{1} B_{2}$, soweit er im Feldinneren verläuft, mit einen Extremalenbogen $A_{1} A_{2}$ zusammenfallen muss, an den

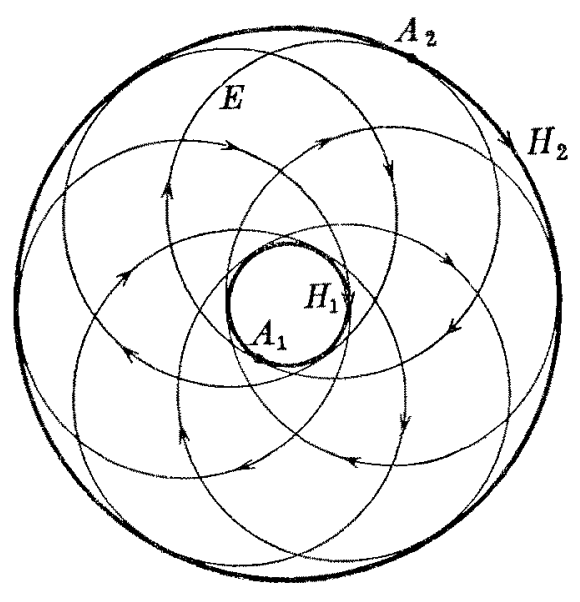

Fig. 3 . 
sich vor $A_{1}$ und nach $A_{2}$ je ein Bogen der Hülle $H_{1}$ bzw. $H_{2}$ anschliessen kann. ("verlängerter» Extremalenbogen). Analoges gilt für den Bogen $B_{2} B_{1}$ : Demnach gilt das Gleichheitszeichen in (2) nur dann, wenn $C$ Feldextremale ist oder sich aus zwei «verlängerten Extremalenbogen » zusammensetzt, also eine "verlängerts Extremale» darstellt, die aus zwei Bogen $A_{1} A_{2}$ und $A_{2}^{\prime} A_{1}^{\prime}$ geschlossener Feldextremalen unter $Z$ wischenschaltung zweier Stüchke von $H_{1}$ bzw. $H_{2}$ zusammensetz (vgl. Fig. 2). Solche verlängerte Extremalen gibt es offenbar nur dann, wenn sich bei Verschiebung von $A_{1}$ im positiven Sinn längs $H_{1}$ im negativen Sinn längs $H_{2}$ bewegt; in dem durch Fig. 3 versinnlichten umgekehrten Fall gilt daher stetz $J_{C}>J_{E}$, wenn $C$ nicht Feld. extremale ist.

\section{II.}

1. Wir betrachten nun den Sonderfall :

$$
F=\lambda \sqrt{\dot{x}^{2}+\dot{y}^{2}}-\frac{1}{2}(x y-y \dot{y}),
$$

wo $\lambda$ eine positive Konstante ist. Die Extremalen sind hier die positiv umlaufenen Kreise vom Radius $\lambda$, also alle geschlossen, das Integral $J$ hat für jede derselben den Wert $\pi \lambda^{2}$.

Sei nun $C$ eine geschlossene Kurve, die in einem von zwei parallelen Geraden begrenzten Streifen der Breite $2 \lambda$ verläuft und beide Ründer des Streifens trifft. Die dem Streifen einbeschriebenen Kreise bilden ein Feld im Sinn von I, 2. Daher liefert das Ergebnis vom I, 2 sofort

$$
\lambda L_{C}-F_{C} \geqq \pi \lambda^{2},
$$

dann unser Variationsproblem ist positiv-regulär, sodass alle Extremalen stark sind. Hieraus schliesst man aber bekanntlich sofort anf die «isoperimetrische Ungleichung», indem man bemerkt, dass das quadratische Polynom in $\lambda$ :

$$
\pi \lambda^{2}-\lambda L_{0}
$$

wegen (3) Werte $<0$ annimmt. Bei dem Beweis werden keine Voranssetzungen über Konvexität benötigt, man kommt, wie eine eingehendere Betrachtung leicht ergibt, mit der einzigen Voraussetzung der Rektifizierbarkeit von $C$ aus.

2. Statt der einem Parallelstreifen eingeshriebenen Kreise kann man natürlich andere Kreisscharen benützen, z. B. Kreise, deren Mittelpunkte auf einem festen Kreis liegen. Von Interesse ist der folgende Spezialfall : Kreise vom Radius $\lambda>0$, deren Mittelpunkte auf einem Kreis vom Radius $\mu<\lambda$ liegen. Durch diese Schar wird ein Kreisring mit dem änsseren Radius $\lambda+\mu$, dem inneren $\lambda-\mu$ doppelt überdeckt. Hier liegt der in Fig. 3. dargestellte Fall vor. Es erhellt, dass für jede geschlossene, positiv umlaufene Kurve, die 
im Ringgebiet einen einfachen Umlauf macht und beide Ränder trifft, wieder die Ungleichung

$$
\lambda L-F \geqq \pi \lambda^{2}
$$

gilt, in der nur für die feldbildenden Kreise das Gleichheitszeichen eintritt. Hieraus folgt die Ungleichung:

$$
\frac{L-\sqrt{L^{2}-4 \pi F}}{\pi} \leqq 2 \lambda \leqq \frac{L+\sqrt{L^{2}-4 \pi F}}{\pi}
$$

Insbesondere gilt für den inneren und äusseren Radins des Minimalkreisrings $\left({ }^{2}\right)$ die entsprechende Ungleichung:

$$
\frac{L-\sqrt{L^{2}-4 \mu F}}{\pi} \leqq p+P \leqq \frac{L+\sqrt{L^{2}-4 \pi F}}{\pi} .
$$

3. Die Betrachtungen dieses Abschnittes gestatten ebenso den Beweis der isoperimetrischen Ungleichung für die elliptisehe und die hyperbolische Ebene. Man verwendet dabei wieder Scharen von Kreisen mit festem Radins, deren Mittelpunkte auf einer Geraden liegen. Ebenso ist es möglich, Minkowskis Ungleichung für den gemischten Inhalt ebener konvexer Bereiche mit unserer Methode heruleiten und dabei auch eine Erweiterung auf den Fall zu erhalten, dass einer der Bereiche nicht konvex ist.

\section{III.}

1. Man kann die Betrachtungen von I auf Doppelintegrale mit geschlossenen Extremalen von Kugeltypus übertragen. Man exhält dann eine Aussage uber das Extrem, wobei als konkurrierende Flächen soiche zugelassen sind, die im einer eine Schar geschlossener Extremalen einhüllenden Rôhre verlaufen und deren Mantel längs einer die Röhre umschlingenden Kurve berühren. Wendet man das auf ein Variationsproblem an, unter dessen Extremalen die Kugeln auftreten, so erhält man bei Benutzung einer Schar von Kugeln, deren Mittelpunkte auf einer Geraden liegen, insbesondere Aussagen über die dem Hüllzylinder eingeschriebenen Drehflächen. Mit Hilfe der Verkreisung von H. A. ScrWARz $\left(^{2}\right)$ kann man dann auf bekannte Weise z.B. die Minimaleigenschaft der Kugel beweisen. Statt dies näher auszuführen, will ich lieber zeigen, wie dieser Gedanke eine ganz elementare, die Mittel der Variationsrechnung nicht explizit heranziehende Methode zum Beweis der « isepiphanen Ungleichung» für Drehflächen liefert.

(2) Vgl. Bonnesen-Fenchex, Theorie der konvexen Korper, Berlin 1984, S. 35.

(3) Ebenda, S. 71 f. 
2. Wir betrachten eine ebene Kurve $C$ :

$$
x=x(t), \quad y=y(t) ; \quad 0 \leqq t \leqq 1,
$$

die ganz in $y \geq 0$ verliaft und ihre beiden Endpunkte anf der $x$-Achse hat, Das Maximum von $y(t)$ sei $\lambda>0$ und werde fur $t=\tau$ angenommen $(0<\tau<1)$. Den Teilbogen für $0 \leqq t \leqq \tau$ nennen wir $C_{1}$, den Bogen $\tau \leqq t \leqq 1 C_{2}$.

Aus der Ungleichung von CAUCHY-ScHWarZ folgt sofort:

$$
\left(\lambda \sqrt{\dot{x}^{2}+\dot{y}^{2}}-y \dot{x}\right) y \geqq \sqrt{\lambda^{2}}=y^{2} y y \quad\left(\sqrt{\lambda^{2}-y^{2}} \geqq 0\right)
$$

und das Gleichheitszeichen gilt dabei nur, wenn:

$$
y=0 \quad \text { oder } \quad \dot{x}=k y, \dot{y}=k \sqrt{\lambda^{2}-y^{2}} \quad(k>0) .
$$

Durch Integration ergibt sich:

$$
\lambda \int_{0}^{\tau} y \sqrt{\dot{x}^{2}+\dot{y}^{2}} d t-\int_{0}^{\tau} y^{2} \dot{x} d t \geqq \int_{0}^{\tau} \sqrt{\lambda^{2}-y^{2}} y \dot{y} d t=\int_{0}^{\lambda} y \sqrt{\lambda^{2}-y^{2}} d y=\frac{\lambda^{3}}{3},
$$

Verfährt man analog mit der durch Vorzeichenwechsel der rechten Seite von (4) entstehenden Ungleichung, so folgt weiter:

$$
\lambda \int_{\tau}^{1} y \sqrt{\dot{x}^{2}+\dot{y}^{2}} d t-\int_{\tau}^{1} y^{2} \dot{x} d t \geqq-\int_{\tau}^{1} \sqrt{\lambda^{2}-y^{2}} y y d t=-\int_{\lambda}^{0} y \sqrt{\lambda^{2}-y^{2}} d y=\frac{\lambda^{3}}{3} .
$$

Addition ergibt dann, wenn noch mit $2 \pi$ multipliziert wird:

$$
\lambda O-2 V \geqq \frac{4 \lambda^{3} \pi}{3},
$$

wo $O$ die Oberfläche, $V$ das Volumen der von $C$ bei Drehung um die $x$-Achse erzengten Rotationsfläche bedeutet. Da das Polynom in $\lambda$ :

$$
\frac{4 \lambda^{3} \pi}{3}-\lambda .0
$$

fur $\lambda \geq 0$ den Minimalwert $\frac{1}{6 \sqrt{\pi}} O^{3}$ annimmt, ergibt sich:

$$
O^{3} \geqq 36 \pi V^{2}
$$

Das Gleichheitszeichen kann nur eintreten - dies zeigt eine Betrachtung, die ganz analog zu der in I, 2 durchgefürten ist - wenn $C$ sich aus zwei Viertelkreisen unter Einschub eines Stückes von $y=1$ (das auch wegfallen kann) zusammensetzt (Stücke ron $y=0$, die zunächst auch auftreten können, sind fur das Problem ohne Bedentung). Will man jetzt zur isepiphanen Ungleichung für beliebige Flächen gelangen, so brancht man in bekannter Weise nur die Verkreisung von Sorwanz zu benutzen. Walls die betrachtete Fläche keine Kugel ist, so wird bei Verkreisung mit geeigneter Achse sicher $O$ verkleinert und damit die Ungleichung (6) automatisch zu

$$
O^{3}>36 \pi V^{2}
$$

verschärft, sodass man sich die Diskussion der Frage, wann in (5) das Gleichheitszeichen gilt, uberhaupt ersparen kann. 